\title{
Sinusoidal Response of $d c$ SQUIDs For rf Power Measurements
}

\begin{abstract}
Robert L. Peterson
National Bureau of Standards Boulder, CO 80303
\end{abstract}

Current, power, and attenuation measurements with rf SQUIDs are based on the fact that the voltage from the microwave readout circuit can be made a nearly sinusoidal function of the magnetic flux threading the SQUID. We point out here that an asymmetric dc SQUID with sufficiently low inductance can achieve a very sinusoidal output voltage with good modulation depth. The spectral purity of the sinusoid can be substantially better than that obtained with rf SQUID systems. The purity improves with increasing asymmetry of the junction critical currents, and decreasing values of the $L I_{c}$ product, where $L$ is the SQUID inductance and $I_{c}$ is the smaller of the critical currents. Results of several calculations are presented. Substantial improvement in SQUID methods of if current, power, or attenuation measurement may thus be possible with use of such dc SQUIDs.

Key words: attenuation; rf-measurements; SQUID; superconductivity.

Accepted: January 28, 1987

\section{Introduction}

In the early 1970s, Kamper et al. [1-5] ${ }^{1}$ recognized that superconducting quantum interference devices (SQUIDs), with their periodic response to magnetic flux $\Phi$, had the potential for being used in a completely new kind of current, power, or attenuation measurement. In the systems developed, the rf impedance of a single-junction SQUID ( $\mathrm{rf}$ SQUID) was measured. The impedance variations were reflected into a resonant circuit and measured as variations in phase or amplitude of the output if voltage. If the voltage response is purely sinusoidal in flux with period equal to the flux quantum $\Phi_{0}=h / 2 e$,

About the Author: Robert L. Peterson serves with the Electromagnetic Technology Division in the Center for Electronics and Electrical Engineering, NBS.

\footnotetext{
${ }^{1}$ Numbers in brackets indicate literature references.
}

$$
V=V_{0} \cos \left(2 \pi \Phi / \Phi_{0}\right),
$$

the time-averaged voltage response to a sinusoidally varying flux of amplitude $\Phi_{m}$ takes the form

$$
\bar{V}=V_{0} J_{0}\left(2 \pi \Phi_{m} / \Phi_{0}\right) .
$$

Equation (1) is achieved approximately with use of an overdamped SQUID and a broadband microwave readout circuit [4]. By determining the zeroes of the response experimentally as the flux amplitude is varied, and equating them to the zeroes of the Bessel function $J_{0}$, one can determine $\Phi_{m}$ or its equivalent in terms of current.

The periodic response to an impressed flux is, however, not ideally sinusoidal, and this necessitates an elaborate series of corrective procedures [2-7]. The purpose of this note is to point out that a dc SQUID (a superconducting loop containing 
two Josephson junctions-see fig. 1) with asymmetric critical currents and sufficiently small inductance and capacitance can have a considerably purer sinusoidal voltage output with adequate modulation. Thus one of the major sources of difficulty in realizing a Josephson junction device for $\mathrm{rf}$ measurements can potentially be removed. Although the SQUID inductance is small, the current in the input coil necessary to couple flux into the SQUID can be kept at a reasonable level. The noise in such SQUIDs is also expected to be small.

The threshold characteristic (i.e., maximum zerovoltage current $v$ s. flux) of a symmetric dc SQUID is not at all sinusoidal. Figure 2 shows an example for a 4-pH SQUID with equal critical currents of $55 \mu \mathrm{A}$. It has long been known, however, that the

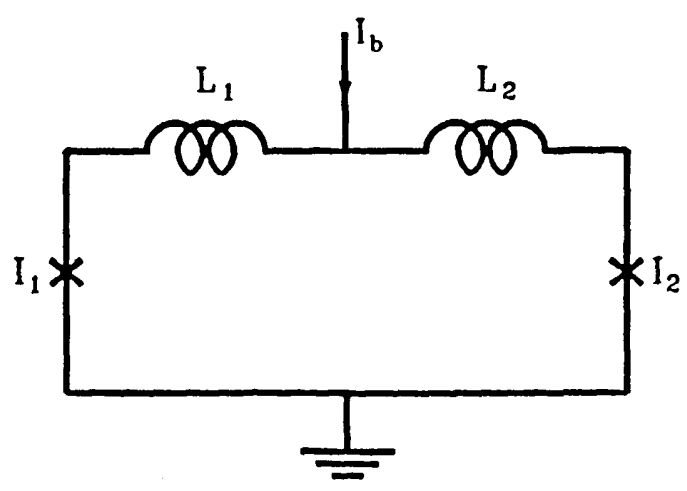

Figure 1-Diagram of a de SQUID with bias current. The inductances and junctions on the two sides may be different. The crosses represent the junctions, including resistance and capacitance. voltage developed across a symmetric SQUID has a sinusoidal appearance when the bias current is sufficiently greater than the maximum critical current of the device. This is observed experimentally and from calculations (see, e.g., [8] and references therein).

That an asymmetric dc SQUID can exhibit a sinusoidal threshold characteristic has been noted earlier by us [9] as well as by Fulton et al. [10]. No specific application was seen at the time. Intuition suggests, however, that this sinusoidal character should be reflected in the voltage developed across the SQUID, and calculations bear this out, as we shall presently show.

The equations describing the threshold behavior of a dc SQUID are [9]

$$
\begin{aligned}
& \cos \phi_{2}=-\cos \phi_{1} /\left(\alpha+\beta \cos \phi_{1}\right), \\
& I_{m}=I_{01} \sin \phi_{1}+I_{02} \sin \phi_{2}, \\
& 2 \pi \Phi / \Phi_{0}=\phi_{2}-\phi_{1}+\beta_{2} \sin \phi_{2}-\beta_{1} \sin \phi_{1},
\end{aligned}
$$

where $\phi_{1}$ and $\phi_{2}$ are the quantum mechanical phase differences across the two Josephson junctions, $I_{01}$ and $I_{02}$ are the respective critical currents, $\alpha=I_{02} /$ $I_{01}, \beta_{i}=2 \pi L_{i} I_{0 i} / \Phi_{0}, \beta=2 \pi L I_{02} / \Phi_{0}, L=L_{1}+L_{2}$, and $L_{1}$ and $L_{2}$ are the lumped inductances for the two segments of the SQUID. See figure 1. Equation (3) shows that there may not be a real solution for $\phi_{2}$ if the denominator on the right is less than unity. In fact if $\alpha$ and $\beta$ are each much less than unity, $\phi_{2}$ has

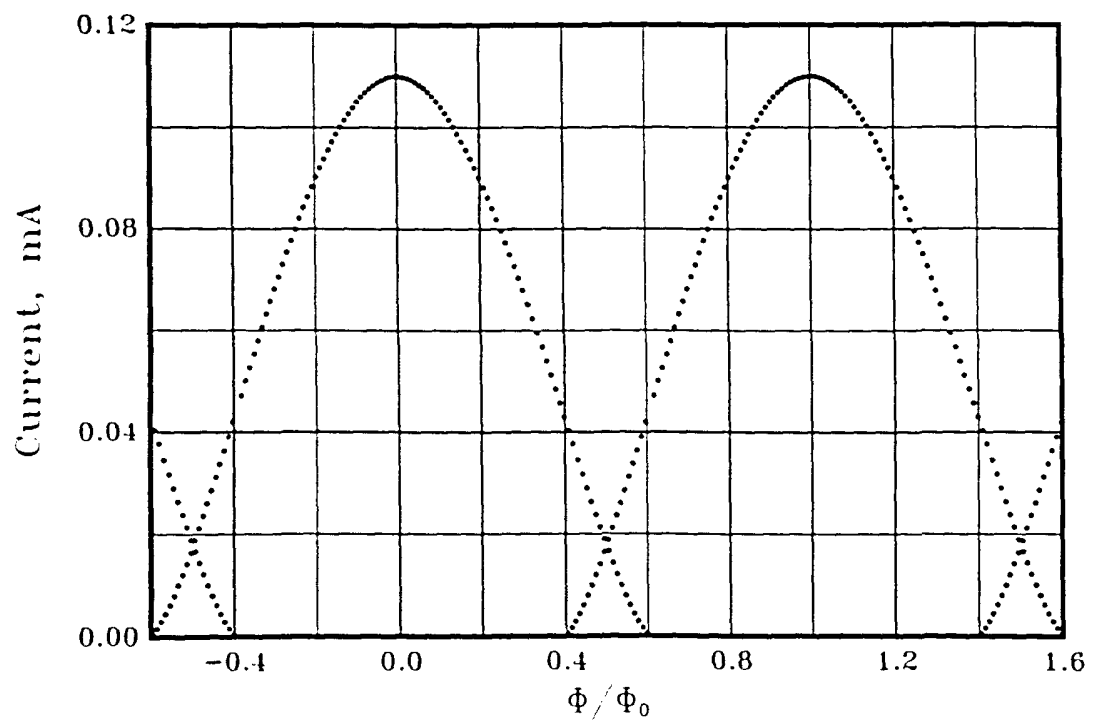

Figure 2-The positive half of the threshold characteristic of a symmetric dc SQUID having $L_{1}=L_{2}=2.0 \mathrm{pH}$ and $I_{01}=I_{02}=$ $55 \mu \mathrm{A}$. 
a solution only for $\phi_{1}$ very close to $(2 n+1) \pi / 2$ where $n$ is any integer or zero. Choosing $\phi_{1} \approx \pi / 2$ and noting that the requirements of $\alpha \ll 1$ and $\beta \ll$ 1 requires $\beta_{2} \ll 1$ (although $\beta_{1}$ may still be of the order of unity), we find from eqs (4) and (5) that

$$
\begin{aligned}
I_{m} & \approx I_{01}+I_{02} \sin \left(2 \pi \Phi / \Phi_{0}-\beta_{2} \sin \phi_{2}+\pi / 2+\beta_{1}\right) \\
& \approx I_{01}+I_{02} \cos \left(2 \pi \Phi / \Phi_{0}+\beta_{1}\right) .
\end{aligned}
$$

This establishes the approximate sinusoidal behavior of the threshold characteristic of a sufficiently asymmetric dc SQUID with low inductance.

In figure 3 we show the computed threshold behavior of an asymmetric dc SQUID, having equal inductances $L_{1}=L_{2}=2.0 \mathrm{pH}$, and unequal critical currents $I_{01}=100 \mu \mathrm{A}, I_{02}=10 \mu \mathrm{A}$. We also plot the perfect sinusoid of eq (6) for comparison. Calculations show that the spectral purity of the threshold characteristic can be improved by making the inductances asymmetric as well. This remarkable purity of the threshold characteristic is carried over into the voltage across the SQUID.

The dynamic equations describing a dc SQUID are

$$
\begin{aligned}
& L_{1} C_{1} \frac{d^{2} \phi_{1}}{d t^{2}}-L_{2} C_{2} \frac{d^{2} \phi_{2}}{d t^{2}}+\frac{L_{1}}{R_{1}} \frac{d \phi_{1}}{d t}-\frac{L_{2}}{R_{2}} \frac{d \phi_{2}}{d t} \\
& +\phi_{1}-\phi_{2}+\beta_{1} \sin \phi_{1}-\beta_{2} \sin \phi_{2}+2 \pi \frac{\Phi}{\Phi_{0}}=0 \\
& C_{1} \frac{d^{2} \phi_{1}}{d t^{2}}+C_{2} \frac{d^{2} \phi_{2}}{d t^{2}}+\frac{1}{R_{1}} \frac{d \phi_{1}}{d t}+\frac{1}{R_{2}} \frac{d \phi_{2}}{d t}
\end{aligned}
$$

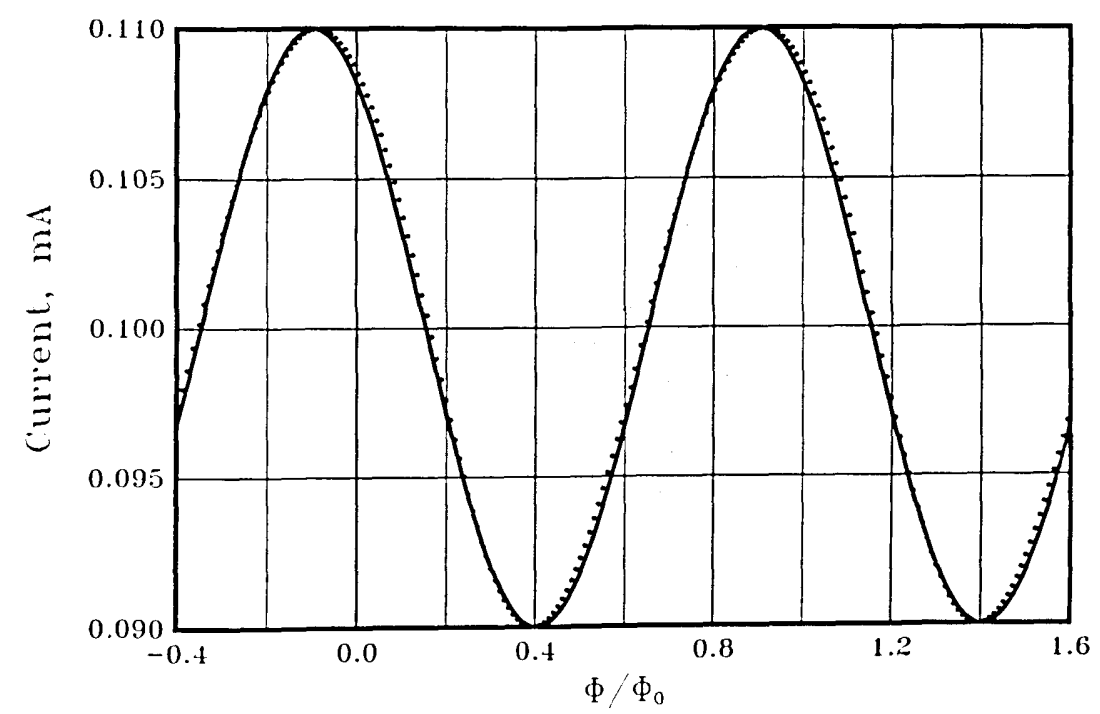

Figure 3-The positive portion of the threshold characteristic of an asymmetric dc SQUID with $L_{1}=L_{2}=2.0 \mathrm{pH}, I_{01}=100 \mu \mathrm{A}$, $I_{02}=10 \mu \mathrm{A}$. The solid line is the sinusoid of eq (6) with $\beta_{1}=0.61$. 


\section{Journal of Research of the National Bureau of Standards}
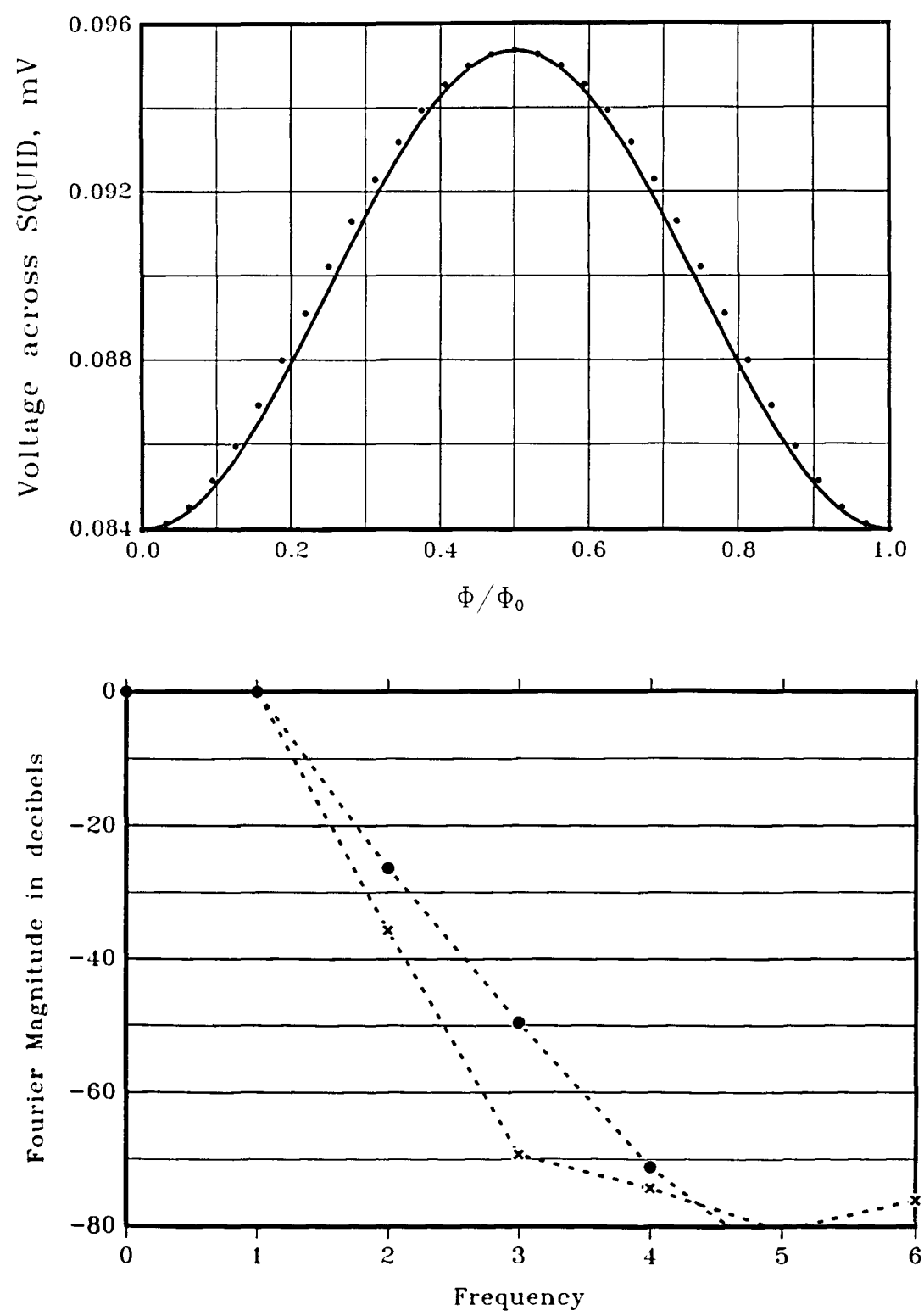

Figure 4-The time-averaged voltage across the symmetric SQUID of figure 2 with a current bias of $200 \mu \mathrm{A}$ (dots). Shunt resistances of $1 \Omega$ and junction capacitances of $0.66 \mathrm{pF}$ are used. The solid line is a sinusoid for visual comparison.

Figure 5-Frequency spectra, in decibels, of the time-averaged voltage across two dc SQUIDs. The solid circles represent the symmetric SQUID of figures 2 and 4 . The crosses represent the asymmetric SQUID of figures 3 and 6. The two SQUIDs have the same total inductance and critical current. Levels below about $-70 \mathrm{~dB}$ are significantly affected by computational noise (see text).

about $-70 \mathrm{~dB}$ in figure 5 are significantly affected by computational noise.

The solid circles in figure 5 show the spectrum of the symmetric SQUID of figure 4. The spectral purity of the voltage of this SQUID is better than that reported for an rf SQUID [4]. The second harmonic is about $26 \mathrm{~dB}$ below ( 5 percent of) the fundamental, and the third harmonic is $50 \mathrm{~dB}$ down ( 0.3 percent).

Figure 6 shows the time-averaged voltage $v s$. flux for the asymmetric SQUID of figure 3, again with a bias current of $200 \mu \mathrm{A}$ and shunt resistances of $1 \Omega$. The junction capacitances are calculated as above and have values of 1.2 and $0.12 \mathrm{pF}$, corresponding to critical currents of $100 \mu \mathrm{A}$ and $10 \mu \mathrm{A}$. The calculations show an excellent voltage sinusoid with a voltage modulation of $4 \mu \mathrm{V}$ relative to an average voltage of about $83 \mu \mathrm{V}$, or about 5 percent modulation. A perfect sinusoid is also shown for visual comparison. The frequency spectrum of this SQUID is shown as the crosses in figure 5. The improvement in spectral purity of the asymmetric SQUID over the symmetric SQUID with the same total inductance and critical current is substantial. The second and third harmonics are now down 36 and $69 \mathrm{~dB}$. The depth of modulation 


\section{Journal of Research of the National Bureau of Standards}

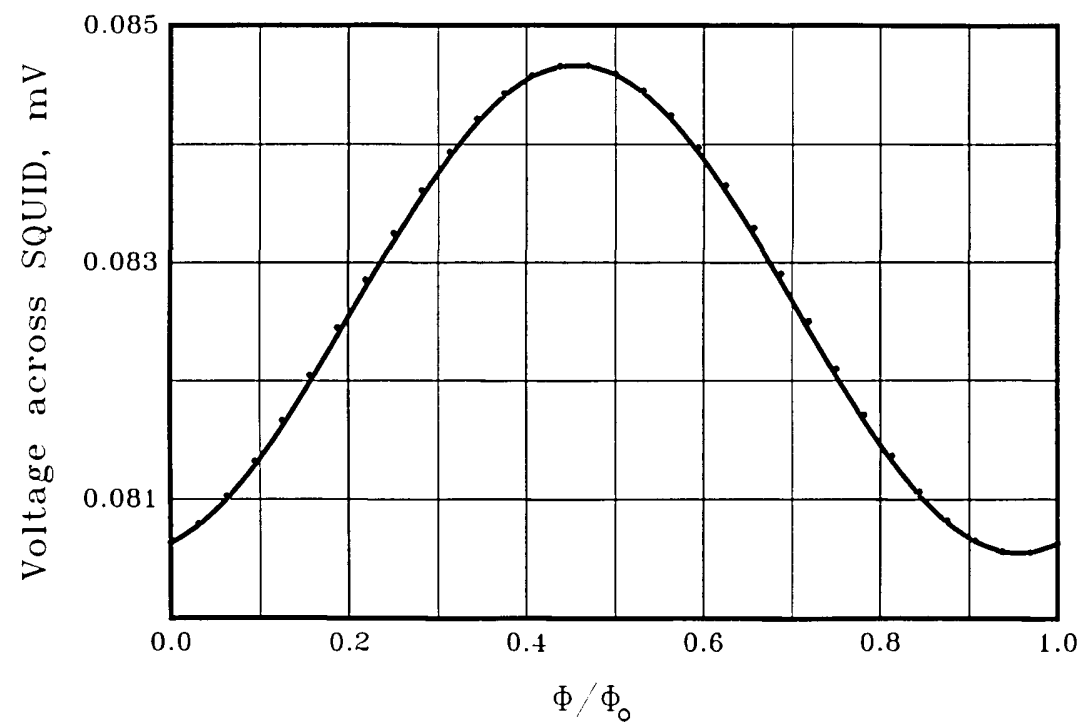

Figure 6-The time-averaged voltage across the asymmetric SQUID of figure 3 with a current bias of $200 \mu \mathrm{A}$ (dots). Shunt resistances of $1 \Omega$ each are used. Junction capacitances are $C_{1}=1.2 \mathrm{pF}$ and $C_{2}=0.12 \mathrm{pF}$. The solid line is a sinusoid for visual comparison.

of the voltage is not as large as with the symmetric SQUID, however. We find this to be generally true - asymmetric SQUIDs have superior spectral purity but less depth of modulation than symmetric SQUIDs with the same bias current and the same total inductance, critical current, and shunt resistance.

The computed I-V curves of figures $7(a, b)$ show the differences in modulation for the two SQUIDs considered here. Curves are shown for $\Phi=0$ and

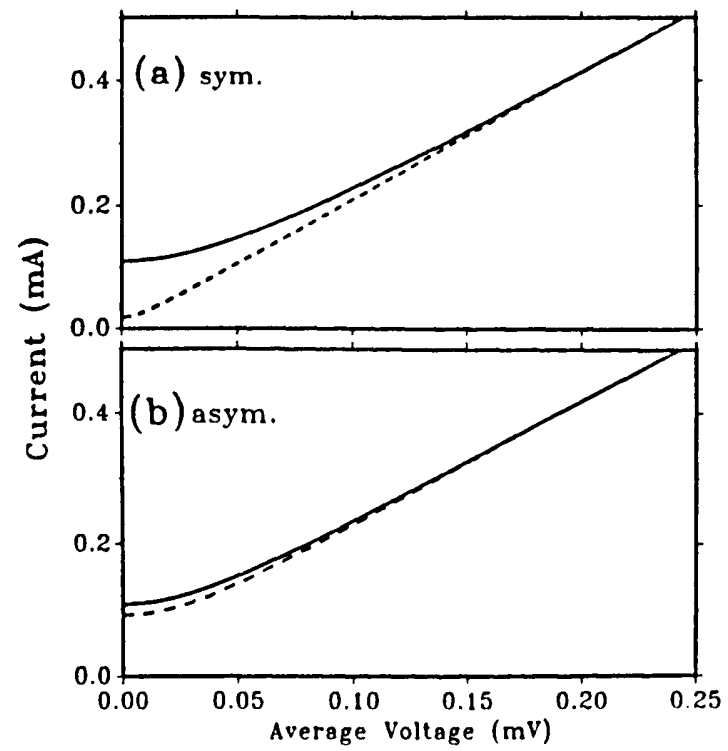

Figure 7-Current-voltage characteristics for dc SQUIDs at $\Phi=0$ (solid curves) and $\Phi=0.5 \Phi_{0}$ (dashed curves). $L_{1}=L_{2}=2.0 \mathrm{pH}$ and $R_{1}=R_{2}=1 \Omega$ for both cases. (a) Symmetric SQUID with $I_{01}=I_{02}=55 \mu \mathrm{A}, C_{1}=C_{2}=0.66 \mathrm{pF}$. (b) Asymmetric SQUID with $I_{01}=100 \mu \mathrm{A}, I_{02}=10 \mu \mathrm{A}, C_{1}=1.2$ $\mathrm{pF}, C_{2}=0.12 \mathrm{pF}$.
$\Phi=0.5 \Phi_{0}$. Note that these values of flux are not quite at the extrema of the voltage for the asymmetric SQUID, as figure 6 shows. Spectral purity generally increases as the bias current is increased, but at the expense of depth of modulation. An exception occurs when an LC resonance significantly affects the current-voltage relation, as shown in figure 8. Here the shunt resistances are kept at $1 \Omega$ but the critical currents, and hence capacitances, of the SQUIDs considered above are doubled, and

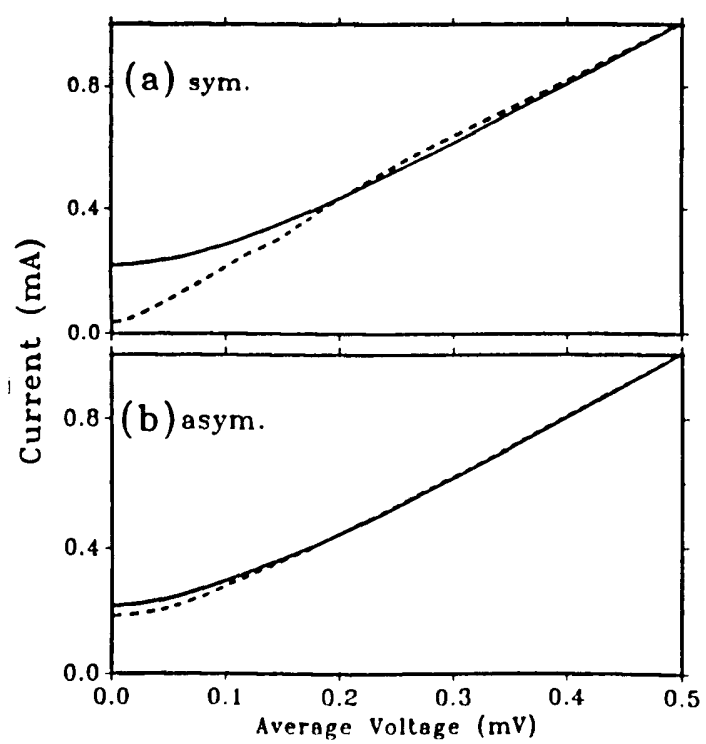

Figure 8-Current-voltage characteristics for dc SQUIDs at $\Phi=0$ (solid curves) and $\Phi=0.5 \Phi_{0}$ (dashed curves). $L_{1}=L_{2}=1.0 \mathrm{pH}$ and $R_{1}=R_{2}=1 \Omega$ for both cases. (a) Symmetric SQUID with $I_{01}=I_{02}=110 \mu \mathrm{A}, C_{1}=C_{2}=1.32 \mathrm{pF}$. (b) Asymmetric SQUID with $I_{01}=200 \mu \mathrm{A}, I_{02}=20 \mu \mathrm{A}, C_{1}=2.4$ $\mathrm{pF}, C_{2}=0.24 \mathrm{pF}$. 
the inductances are halved to keep the $L I_{\mathrm{c}}$ product unchanged. For the symmetric SQUID-figure $8\left(\right.$ a)-an LC resonance at $\Phi_{0} /\left(2 \pi \sqrt{L_{1} C_{1}}\right)=0.29$ $\mathrm{mV}$ is evident. (The resonance becomes sharper with larger values of shunt resistance.) Calculations for SQUIDs with apparent LC resonances show that the spectral purity of the voltage vs. flux curves is degraded. The asymmetric SQUID of figure 8(b) does not show a prominent LC resonance because the two sides of the SQUID interfere; however, the larger capacitance of this example greatly reduces the modulation depth. It is thus important that the junction capacitance be kept as low as possible so that the capacitive impedance does not shunt out the resistance.

Perhaps the principal virtue of the low-inductance SQUIDs, and especially the asymmetric lowinductance SQUIDs, lies in the fact that the harmonics above the second are very greatly reduced and are probably negligible. This is important because the earlier work with rf SQUIDs [5] found that the second harmonic could effectively be nulled, and that the higher harmonics constituted the principal problem. That the second harmonic is also substantially reduced is of course an improvement. Since the basic source of systematic error in the measurement of $\mathrm{rf}$ attenuation is caused by harmonic distortion of the sinusoidal response of the system [4], the SQUIDs discussed here should have a distinct advantage over the $\mathrm{rf}$ SQUIDs.

In $1982 \mathrm{M}$. Cromar of this laboratory made a preliminary study on the suitability of a thin-film dc SQUID as the detector element in an rf attenuator-calibrator system (unpublished). Using a resistively shunted symmetric SQUID whose $L I_{\mathrm{c}}$ product was considerably larger than $\Phi_{0}$, he showed that at low signal frequencies, the zeroes of the response approximated the zeroes of the $J_{0}$ Bessel function closely enough that accuracy at $\mathrm{rf}$ frequencies comparable to that of the earlier measurements with if SQUIDs might be realized. This research was not pushed further.

The microfabricated dc SQUIDs are expected to have better thermal and temporal stability than the single-junction rf SQUIDs used earlier. The overall circuitry necessary to attain the output voltage should also be simpler with the dc SQUID. Imperfections in microwave components were found to be a major contributor to the harn onic distortion in the if SQUID system [5]. Such components would not be used in the readout scheme for a dc SQUID.

The 4-pH inductances used here are small, but SQUIDs with lower inductance have been fabri- cated. The low inductance of the proposed SQUIDs presents a potential problem of sufficient coupling, however. For example, if the mutual inductance between a 4-pH SQUID and the input line were only 4-pH, about $500 \mu \mathrm{A}$ would be required in the line to produce one flux quantum in the SQUID. If one desires 200 nulls in the voltage response (100 flux quanta), about $50 \mathrm{~mA}$ must flow in the line in this case. Although higher than typical, this value is still below what a superconducting stripline could support. Higher values of mutual inductance will decrease the maximum current needed. The mutual inductance to an input coil carrying the signal can in fact be made much larger than the self-inductance of the SQUID. Planar coupling to low-noise, low-inductance SQUIDs is the subject of much current research [11-13]. Of course, one may also choose to work with higherinductance SQUIDs, accepting somewhat less spectral purity if the critical currents are kept at the same values, in order to decrease the maximum current needed in the stripline or to decrease the degree of coupling to the SQUID.

The effect of noise is another consideration. It is beyond the scope of this note to undertake a detailed study of noise effects, which depend upon the readout method used. Ideally, of course, it is desirable that the noise of the SQUID plus its readout system be dominated by the intrinsic noise of the SQUID. Readout schemes with this in view are under active investigation; Ketchen [11] discusses several of them. For a readout system operating at a frequency of about $100 \mathrm{kHz}, 1 / f$ noise is not a consideration. However, even at lower frequencies where $1 / f$ noise dominates, a new readout scheme [14] for significantly reducing the noise has been developed.

Properly fabricated dc SQUIDs have the lowest noise figures of any devices [11]. White noise decreases with decreasing inductance, which is favorable for the SQUIDs under consideration. The parameter $2 \pi k T / I_{0} \Phi_{0}$ is sometimes used to characterize the noise. If we take $I_{0}=0.11 \mathrm{~mA}$, which is the average of the critical currents used in the preceding examples, we find the value 0.002 at $4 \mathrm{~K}$ for this noise parameter. The ultra-low-noise SQUIDs operate near this value. The spectral density $S_{V}$ of the voltage fluctuations in a resistor $R$ is given by $4 k T R$. For shunt resistances of about $1 \Omega$, the voltage noise power spectrum is $2 \times 10^{-22} V^{2} / \mathrm{Hz}$ at $4 \mathrm{~K}$. The flux noise density $S_{\Phi}$ is approximately $S_{V} /|\partial V / \partial \Phi|^{2}$. The ultra-low-noise SQUIDs are built to be biased at that value of flux that gives the greatest energy sensitivity, which occurs at the 
largest value of $|\partial V / \partial \Phi|$. However, in the present case the flux will be swept over many periods of $\Phi_{0}$, so that each value of flux contributes almost equally. Since the voltage is nearly sinusoidal, $|\partial V / \partial \Phi|^{2}$ varies almost as sine-squared, which we may replace by $1 / 2$ times an amplitude, to a good approximation. Thus $S_{\Phi} \approx 2 k T R \Phi_{0}^{2} /\left(\pi V_{0}\right)^{2}$, where $V_{0}$ is the voltage amplitude. For the symmetric SQUID of figure $4, V_{0} \approx 5.5 \mu \mathrm{V}$ so that $S_{\Phi} \approx 10^{-13} \Phi_{0}^{2} / \mathrm{Hz}$. These values are not far from values quoted for low-noise SQUIDs [13-15]. Other expressions for the voltage noise power spectrum are available $[14,15]$ which take into account noise contributions from the circulating current in the SQUID. The noise values from these expressions are not significantly different from those calculated above because of the very small inductance. Although these arguments do not establish that noise will not be a significant problem affecting the accuracy of determining the voltage nulls in $\mathrm{rf}$ measurements, they show that the SQUIDs proposed here share characteristics of low-noise SQUIDs, and thus are encouraging.

The purpose of this note has been to suggest a new scheme for $\mathrm{rf}$ measurements using SQUIDs, and to demonstrate its potential advantages. A more extensive analysis of feasibility could include the following: simulations of the effect of noise upon the accuracy with which the zeroes of the response can be determined; studies of the magnitude and effects of the parasitic inductance associated with the shunt resistors; inclusion of the circuit carrying the signal-source, coupling coil, and load-to determine whether the nonlinear impedance reflected into the circuit by the SQUID is important; determination of the optimum choice of inductance, critical currents, asymmetry, and bias current to obtain the best combination of spectral purity and depth of modulation, or in short the greatest accuracy possible.

The considerations presented here suggest that thin-film, low inductance, low critical current, dc SQUIDs, especially those with asymmetric critical currents, would be superior to rf SQUIDs in rf current, power, or attenuation measurements.
This work has benefitted from discussions with several of my colleagues, to whom I express my thanks.

\section{References}

[1] Kamper, R. A., and M. B. Simmonds, Broadband superconducting quantum magnetometer, Appl. Phys. Lett. 20 270-272 (April 1972).

[2] Kamper, R. A.; M. B. Simmonds, C. A. Hoer, and R. T. Adair, Quantum mechanical measurement of if attenuation, in Proc. Appl. Superconductivity Conf. (IEEE Pub. 72CH0682-5-TABSC) 696-700 (May 1972).

[3] — A New Technique for rf Measurements Using Superconductors, Proc. IEEE 61 121-122 (January 1973).

[4] - Measurement of $\mathrm{rf}$ Power and Attenuation Using Superconducting Quantum Interference Devices, Natl. Bur. Stand. Tech. Note 643 (August 1973).

[5] Kamper, R. A.; M. B. Simmonds, R. T. Adair, and C. A. Hoer, Advances in the Measurement of if Power and Attenuation Using SQUIDs, Natl. Bur. Stand. Tech. Note 661 (September 1974).

[6] Petley, B. W.; K. Morris, R. W. Yell, and R. N. Clarke, Moulded microwave SQUID for $\mathrm{rf}$ attenuator calibration, Electron Lett. 12 237-238 (May 1976).

[7] Sullivan, D. B.; R. T. Adair, and N. V. Frederick, rf Instrumentation Based on Superconducting Quantum Interference, Proc. IEEE 66 454-463 (April 1978).

[8] Peterson, R. L., and D. G. McDonald, Voltage and current expressions for a two-junction superconducting interferometer, J. Appl. Phys. 54 992-996 (February 1983).

[9] Peterson, R. L., and C. A. Hamilton, Analysis of threshold curves for superconducting interferometers, J. Appl. Phys. 50 8135-8142 (December 1979).

[10] Fulton, T. A.; L. N. Dunkleberger, and R. C. Dynes, Quantum interference properties of double Josephson junctions, Phys. Rev. B6 855-875 (August 1972).

[11] Ketchen, M. B., dc SQUIDs 1980: The state of the art, IEEE Trans. Magn. MAG-17 387-394 (January 1981).

[12] Jaycox, J. M., and M. B. Ketchen, Planar coupling scheme for ultra low noise de SQUIDs, IEEE Trans. Magn. MAG17 400-403 (January 1981).

[13] Cromar, M. W., and P. Carelli, Low-noise tunnel junction dc SQUIDs, Appl. Phys. Lett. 38(9) 723-725 (May 1981).

[14] Foglietti, V.; W. J. Gallagher, M. B. Ketchen, A. W. Kleinsasser, R. H. Koch, S. I. Raider, and R. L. Sandstrom, Low frequency noise in low-1/f-noise dc SQUIDs, Appl. Phys. Lett. (in press).

[15] Voss, R. F.; R. B. Laibowitz, A. N. Broers, S. I. Raider, C. $\mathrm{M}$. Knoedler, and J. M. Viggiano, Ultra low noise $\mathrm{Nb}$ dc SQUIDs, IEEE Trans. Magn. MAG-17 395-399 (January 1981). 